CLINICAL STUDY

\title{
Reduced serum total osteocalcin is associated with metabolic syndrome in older men via waist circumference, hyperglycemia, and triglyceride levels
}

\author{
Bu B Yeap ${ }^{1,2}$, S A Paul Chubb ${ }^{1,3}$, Leon Flicker ${ }^{1,4}$, Kieran A McCaul ${ }^{4}$, Peter R Ebeling ${ }^{5}$, John P Beilby ${ }^{3}$ \\ and Paul E Norman ${ }^{6}$ \\ ${ }^{1}$ School of Medicine and Pharmacology, University of Western Australia, Perth, Western Australia 6009, Australia, ${ }^{2}$ Department of Endocrinology and \\ Diabetes, Fremantle Hospital, Fremantle, Western Australia 6160, Australia, ${ }^{3}$ PathWest Laboratory Medicine, Fremantle and Sir Charles Gairdner \\ Hospitals, Perth, Western Australia 6009, Australia, ${ }^{4}$ WA Centre for Health and Ageing, Centre for Medical Research, University of Western Australia, \\ Perth, Western Australia 6009, Australia, ${ }^{5}$ Department of Medicine, University of Melbourne, Melbourne, Victoria 3011, Australia and ${ }^{6}$ School of \\ Surgery, University of Western Australia, Perth, Western Australia 6009, Australia \\ (Correspondence should be addressed to B B Yeap who is now at School of Medicine and Pharmacology, Level 2, T Block, Fremantle Hospital, Alma Street, \\ Fremantle, Western Australia 6160, Australia; Email: byeap@cyllene.uwa.edu.au)
}

\begin{abstract}
Objective: Bone-derived undercarboxylated osteocalcin regulates insulin secretion and sensitivity in mice, and reduced serum total osteocalcin (TOC) is associated with diabetes in humans. However, the relationship between TOC levels and other cardiovascular risk factors is uncertain. We sought to determine whether serum TOC is associated with metabolic syndrome and its components in older men.

Design: Cross-sectional analysis from a population-based cohort of men aged $\geq 70$ years.

Methods: Early morning sera were assayed for TOC. Insulin resistance was estimated using a homeostatic model (HOMA2-IR). Metabolic syndrome was defined according to NCEP-ATPIII criteria. Results: TOC was assayed in 4047 men. Men who were not fasting and reported having bone fractures, Paget's disease, or bisphosphonate, glucocorticoid, or warfarin use were excluded, leaving 2765 men with metabolic syndrome present in $797(28.8 \%)$. TOC was inversely associated with waist circumference, glucose, and triglyceride levels and HOMA2-IR (all $P<0.001$ ), and was lower in men with metabolic syndrome (mean \pm s.E.M.: $20.1 \pm 0.4$ vs $21.4 \pm 0.2 \mu \mathrm{g} / \mathrm{l}, P=0.002$ ). In multivariate analysis, men with TOC of 13.25-16.55 and $<13.25 \mu \mathrm{g} / \mathrm{l}$ had 1.5- to 2-fold increased risk of metabolic syndrome compared with men with levels $\geq 30 \mu \mathrm{g} / \mathrm{l}$. TOC remained associated with metabolic syndrome after adjustment for individual components, but not after adjusting for both waist circumference and glucose.

Conclusions: Increased waist circumference, reduced TOC, elevated glucose, and triglyceride levels are inter-related in aging men. Osteocalcin may lie in the causal pathway between central adiposity and insulin resistance. Further research is required to evaluate whether interventions which raise osteocalcin levels might decrease cardiovascular risk.

European Journal of Endocrinology 163 265-272
\end{abstract}

\section{Introduction}

Osteocalcin is secreted by osteoblasts, and undergoes posttranslational modification by $\gamma$-carboxylation, which facilitates its binding to hydroxyapatite and retention in bone (1). Both carboxylated and undercarboxylated forms of osteocalcin are present in the circulation, and serum total osteocalcin (TOC) is a marker of bone turnover $(2,3)$. Recent studies in knockout mice have identified osteocalcin as an endocrine regulator of glucose metabolism, stimulating $\beta$-cell insulin secretion and reducing peripheral insulin resistance (4). In these studies, Esp knockout mice (Esp-/-) lacking an osteoblast-expressed receptor-like protein tyrosine phosphatase (OST-PTP) displayed increased $\beta$-cell proliferation, elevated insulin levels, and lower glucose levels, and were protected from developing diabetes. The diabetes-resistant Esp-/phenotype was completely reversed by the deletion of a single allele of Osteocalcin (4). Ocn - / - mice exhibited lower serum total adiponectin levels and impaired glucose tolerance in vivo, with osteocalcin regulating insulin sensitivity independently of its effect on insulin secretion. Exposure to undercarboxylated osteocalcin (ucOC) increased the expression of adiponectin in adipocytes and insulin in islets, and administration of ucOC to mice resulted in insulin sensitizing effects, indentifying ucOC as metabolically active $(4,5)$. 
There are limited data confirming the role of ucOC as a regulator of insulin secretion and sensitivity in humans. The presence of type 2 diabetes has been associated with lower levels of TOC, which comprises ucOC and carboxylated osteocalcin (6-8). Among diabetic patients, higher TOC levels correlated with lower fasting plasma glucose levels and better glycemic control $(6,9)$. In a 16-week study, marked weight loss achieved by diet resulted in increased serum TOC, as did moderate loss coupled with resistance training (10). Serum TOC correlated inversely with fat mass and body mass index (BMI) (7), and higher TOC was associated with a lower rise in fasting glucose after 3 years (11). These data could be interpreted as suggesting obesity, diabetes, or higher glucose levels are associated with reduced bone formation, which might explain in part the higher risk of hip fracture seen in men with type 2 diabetes despite preserved bone density (12).

While cardiovascular disease is the major cause of morbidity and premature mortality in persons with type 2 diabetes (13-16), it remains unclear whether circulating osteocalcin, as a consequence or otherwise of its association with diabetes, might influence the risk of cardiovascular disease in humans. As older men are at risk of diabetes and cardiovascular diseases and have impaired bone strength resulting in increased incidence of bone fracture (17), the role of osteocalcin could be particularly relevant in this setting. Therefore, we sought to determine whether reduced TOC levels were associated with risk factors for cardiovascular disease, as reflected by the presence of metabolic syndrome, in community-dwelling older men. In addition, we sought to determine whether this association was predominantly due to an association between lower serum TOC and hyperglycemia, or whether lower TOC level was associated with other metabolic risk factors.

\section{Participants and methods}

\section{Study population}

Details of the Health In Men Study (HIMS) have been described in depth elsewhere (18). Briefly, between October 2001 and August 2004, a population-based sample of 4263 community-dwelling men resident in metropolitan Perth, Western Australia, participated in the study. Men were predominantly of Caucasian ethnicity. Demographic, medical, and medications data were collected. Height (in centimeters), weight (in kilograms), waist and hip circumference (in centimeters), and blood pressure were measured using standard procedures. An early morning blood sample was collected for analysis of biochemistry and hormone levels. The human research ethics committee of the University of Western Australia approved the study protocol, and all study participants gave their written informed consent.

\section{Definition of metabolic syndrome}

Metabolic syndrome was defined according to the NCEP-ATPIII classification as three or more of waist circumference $>102 \mathrm{~cm}$, fasting plasma glucose $\geq 5.6 \mathrm{mmol} / \mathrm{l}$, or a known diagnosis of diabetes, fasting serum triglycerides $\geq 1.7 \mathrm{mmol} / \mathrm{l}$, fasting high-density lipoprotein (HDL) cholesterol $<1.03 \mathrm{mmol} / \mathrm{l}$, or blood pressure $\geq 130 / 85 \mathrm{mmHg}$ (19). We used the recent modification of the definition, which lowered the threshold for an abnormal glucose concentration to $\geq 5.6 \mathrm{mmol} / \mathrm{l}$ (20). Men with a previous diagnosis of hypertension or taking anti-hypertensive therapy were regarded as fulfilling the criterion for hypertension.

\section{Laboratory assays}

Blood samples were collected between 0800 and $1030 \mathrm{~h}$. Serum was prepared immediately following phlebotomy and stored at $-80{ }^{\circ} \mathrm{C}$ until assayed. Biochemical and hormone assays were performed in the Biochemistry Department, PathWest Laboratory Medicine, Fremantle and Royal Perth Hospitals, Western Australia. Serum TOC and insulin were assayed using the automated Elecsys assay (Roche Diagnostics). This TOC assay detects both intact osteocalcin (amino acids 1-49) and the major N-terminal fragment (1-43). The inter-assay coefficients of variation were $3.6 \%$ at 17.1 and $89.6 \mu \mathrm{g} / \mathrm{l}$ for TOC and $4.6 \%$ at $11 \mathrm{mU} / \mathrm{l}$ and $3.3 \%$ at $33 \mathrm{mU} / \mathrm{l}$ for insulin. Fasting serum glucose, total and HDL cholesterol, and triglycerides were estimated using a Roche Hitachi 917 analyser (Roche Diagnostic GmbH). Between-day imprecision for glucose was $2.9 \%$ at $4.8 \mathrm{mmol} / \mathrm{l}$ and $2.2 \%$ at $15.2 \mathrm{mmol} / \mathrm{l}$, for cholesterol it was $2.3 \%$ at $3.2 \mathrm{mmol} / \mathrm{l}$ and $2.1 \%$ at $6.7 \mathrm{mmol} / \mathrm{l}$, for HDL it was $2.4 \%$ at $0.8 \mathrm{mmol} / \mathrm{l}$ and $2.5 \%$ at $1.7 \mathrm{mmol} / \mathrm{l}$, and for triglycerides it was $4.8 \%$ at $0.9 \mathrm{mmol} / \mathrm{l}$ and $2.4 \%$ at $2.0 \mathrm{mmol} / \mathrm{l}$. Insulin resistance was estimated from fasting glucose and insulin results by homeostasis model assessment, using the spreadsheet implementation of the HOMA2 calculator (HOMA2-IR, downloaded from www.dtu.ox.ac.uk/homa) (21).

\section{Statistical analysis}

Data were analyzed with the statistical package Stata, version 10.0 (StataCorp., College Station, Texas, USA 2007). Distributions of serum TOC in men with and without metabolic syndrome were compared. Data are tabulated as mean \pm s.D. or S.E.M. Mean comparisons were performed using Student's $t$-test. To allow analysis of non-parametrically distributed variables, quantile regression was used to model median and 75 th percentile as well as mean for components of metabolic syndrome against TOC concentrations. The association of serum TOC with insulin resistance (HOMA2-IR) was examined in the same manner. Logistic regression 
analysis was used to assess odds ratio (OR) for metabolic syndrome for values of serum TOC adjusting for age, smoking, alcohol use, and serum creatinine. These analyses were repeated including adjustment for each individual component of metabolic syndrome. $P$ values of $<0.05$ were considered significant.

\section{Results}

\section{Distribution of serum TOC in community-dwelling older men}

Of the cohort of 4263 men, aliquots of serum from 4047 men were available for assay of TOC. Men who reported having bone fractures, Paget's disease, or receiving bisphosphonate, glucocorticoid, or warfarin were excluded as these conditions might influence circulating osteocalcin levels $(n=542)$. Following this, men who were not fasting at the time of blood sampling were excluded as no fasting triglyceride or HDL results were available for the classification of metabolic syndrome $(n=740)$. After these exclusions, there were 2765 men with fasting early morning serum TOC results, which were included in the analysis (Fig. 1A). Mean serum TOC was $21.0 \mu \mathrm{g} / \mathrm{l}$, median was $19.0 \mu \mathrm{g} / \mathrm{l}$, and interquartile range was 14.7-24.5 $\mu \mathrm{g} / \mathrm{l}$. Men who were excluded only because they had not fasted had a similar distribution of serum TOC with mean $20.4 \mu \mathrm{g} / \mathrm{l}$, median $18.5 \mu \mathrm{g} / \mathrm{l}$, and interquartile range 14.5-23.7 $\mu \mathrm{g} / \mathrm{l}$ (Fig. 1B).

\section{Stratification of men according to the presence of metabolic syndrome or its components}

Of the final sample of 2765 men, metabolic syndrome was present in $797(28.8 \%)$. Men with metabolic syndrome had higher BMI, waist circumference, systolic and diastolic blood pressure, and triglyceride, glucose, and creatinine levels compared with men without metabolic syndrome (Table 1). Men with metabolic syndrome had lower total, high-, and low-density lipoprotein cholesterol levels and lower serum TOC compared with those who did not have metabolic syndrome. There was no difference in age between the
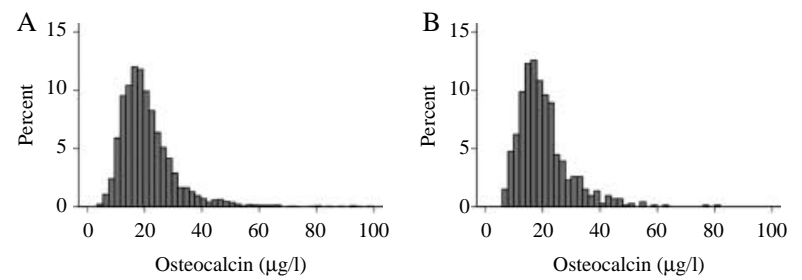

Figure 1 Distribution of serum total osteocalcin in communitydwelling men aged $\geq 70$ years. (A) Serum total osteocalcin in 2765 men who were fasting at the time of blood sampling. (B) Serum tota osteocalcin in 740 men who were not fasting at the time of blood sampling. Osteocalcin units are $\mu \mathrm{g} / \mathrm{l}$.
Table 1 Characteristics of 2765 community-dwelling older men stratified for the absence and the presence of metabolic syndrome. Data are shown as mean (S.E.M).

\begin{tabular}{lrrr}
\hline & $\begin{array}{c}\text { No metabolic } \\
\text { syndrome } \\
(n=1968)\end{array}$ & $\begin{array}{c}\text { Metabolic } \\
\text { syndrome } \\
(n=797)\end{array}$ & $\boldsymbol{P}$ value \\
\hline Age (years) & $76.5(0.08)$ & $76.3(0.12)$ & 0.182 \\
BMl (kg/m $\left.{ }^{2}\right)$ & $25.4(0.07)$ & $29.1(0.12)$ & $<0.001$ \\
Waist $(\mathrm{cm})$ & $95.6(0.19)$ & $106.8(0.30)$ & $<0.001$ \\
Systolic BP $(\mathrm{mmHg})$ & $146.4(0.45)$ & $151.0(0.69)$ & $<0.001$ \\
Diastolic BP $(\mathrm{mmHg})$ & $74.1(0.23)$ & $76.3(0.37)$ & $<0.001$ \\
Creatinine $(\mu \mathrm{mol} / \mathrm{l})$ & $91.3(0.57)$ & $98.2(1.63)$ & $<0.001$ \\
Glucose $(\mathrm{mmol} / \mathrm{l})$ & $5.39(0.02)$ & $6.22(0.05)$ & $<0.001$ \\
TG $(\mathrm{mmol} / \mathrm{l})$ & $1.05(0.01)$ & $1.78(0.03)$ & $<0.001$ \\
HDL (mmol/l) & $1.50(0.01)$ & $1.21(0.01)$ & $<0.001$ \\
LDL (mmol/l) & $2.99(0.02)$ & $2.88(0.03)$ & 0.001 \\
Cholesterol & $4.98(0.02)$ & $4.89(0.03)$ & 0.032 \\
Total osteocalcin $(\mu \mathrm{g} / \mathrm{l})$ & $21.4(0.22)$ & $20.1(0.37)$ & 0.002 \\
\end{tabular}

two groups. Serum TOC levels were highest in men who had zero or one components of metabolic syndrome, and were progressively lower in men with two, three, four, or five components of metabolic syndrome (Fig. 2).

\section{Associations of waist circumference, glucose, and triglyceride levels with serum TOC}

Serum TOC was inversely associated with waist circumference, and with glucose and triglyceride levels (all $P<0.001$ ). Quantile regression to model median and 75 th percentile of these metabolic syndrome components is shown (Fig. $3 \mathrm{~A}-\mathrm{C}$ ). The inverse associations between serum TOC with waist circumference and glucose were apparent only at lower levels of serum TOC $(\leq 20 \mu \mathrm{g} / \mathrm{l})$. The strongest relationship appeared to be between TOC and glucose levels (Fig. 3B), although associations were also present between TOC with waist circumference, and TOC with serum triglycerides (Fig. 2A and C). For TOC levels $>30 \mu \mathrm{g} / \mathrm{l}$, there was little evidence of any strong association, with wider confidence intervals (CI) evident. There were no comparable associations between serum TOC and systolic blood pressure, or osteocalcin and HDL (data not shown).

\section{Serum TOC and insulin resistance in older men}

Serum TOC was inversely associated with insulin resistance, assessed as HOMA2-IR $(P<0.001)$. The inverse relationship between serum TOC and HOMA2-IR was most apparent at lower levels ( $\leq 20 \mu \mathrm{g} / \mathrm{l}$, Fig. 3D), with less evidence of an association and wider CI at higher TOC levels. A similar result was obtained when fasting insulin concentration was plotted against serum TOC. The inverse association of HOMA2-IR with serum TOC seen in the whole cohort (Fig. 3D) was attenuated in men without metabolic syndrome (data not shown). 


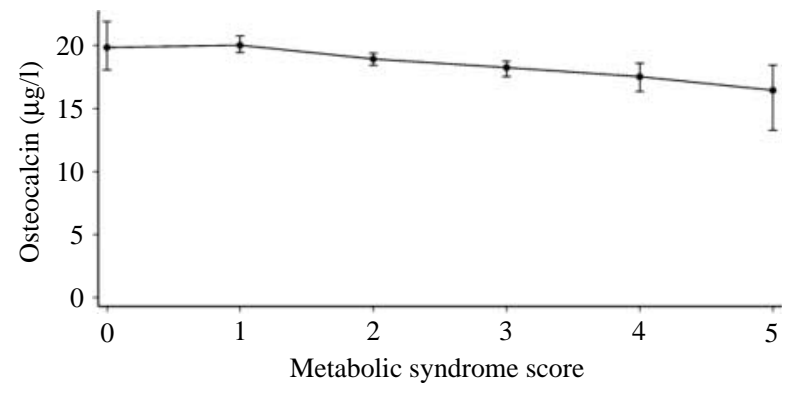

Figure 2 Serum total osteocalcin according to the metabolic syndrome score in 2765 men aged $\geq 70$ years. Metabolic syndrome score is the sum of metabolic syndrome components in each individual man (0-5). Data are median values, and error bars are $95 \%$ confidence intervals.

\section{Serum TOC and metabolic syndrome in older men}

The logistic regression model adjusted for baseline covariates of age, smoking, alcohol intake, and serum creatinine, using men with serum TOC $\geq 30 \mu \mathrm{g} / \mathrm{l}$ as the reference group and stratifying men with levels $<30 \mu \mathrm{g} / \mathrm{l}$ into quintiles. Men with serum TOC in the lowest two quintiles had increased risk of metabolic syndrome (Fig. 4). OR and 95\% CI for metabolic syndrome were $2.39(1.71-3.35)$ for men with serum TOC $<13.25 \mu \mathrm{g} / \mathrm{l}$ and $1.51(1.07-2.13)$ for those with serum TOC between 13.25 and $16.55 \mu \mathrm{g} / \mathrm{l}$.

\section{Associations between serum TOC and metabolic syndrome adjusting for its individual components}

Men with serum TOC $<13.25 \mu \mathrm{g} / \mathrm{l}$ had OR 1.90 (95\% CI 1.27-2.87) for metabolic syndrome after adjusting for baseline covariates as above and also for waist circumference (Fig. 5A). Men with serum TOC $<13.25 \mu \mathrm{g} / \mathrm{l}$ had increased OR of metabolic syndrome after adjustment for baseline covariates and glucose with OR 1.55 (1.06-2.26; Fig. 5B) and after adjustment for baseline covariates and triglyceride level with $\mathrm{OR}$ 1.79 (1.21-2.65; Fig. 5C). The relationship between serum TOC and risk of metabolic syndrome was not attenuated by inclusion of either HDL or systolic blood pressure into the multivariate model (data not shown). Simultaneous inclusion of waist circumference and glucose level into the multivariate model abrogated the association between serum TOC and metabolic syndrome (Fig. 5D).

\section{Discussion}

Studies in knockout and wild-type mice implicated bone-derived circulating ucOC as an endocrine factor stimulating $\beta$-cell insulin secretion and enhancing insulin sensitivity $(4,5)$. Thus, if these mechanisms are relevant in humans, deficient secretion of osteocalcin would be expected to result in greater insulin resistance (22). Insulin resistance predisposes to type 2 diabetes in the presence of $\beta$-cell dysfunction (23), and underlies the definition of metabolic syndrome, which is a marker for increased risk of cardiovascular disease (24-26). Therefore, we tested the hypothesis that reduced circulating TOC was associated with increased cardiovascular risk defined by the presence of metabolic syndrome in older men. The key findings were that in community-dwelling older men, lower serum TOC was associated with increased risk of metabolic syndrome, and this relationship was mediated by waist circumference, glucose, and triglyceride levels, rather than by HDL concentration or blood pressure.

Previous studies have reported on the relationship between serum TOC and glucose metabolism in smaller cohorts of middle-aged and older women and men. Im et al. studied 339 postmenopausal women (mean age 56.5 years), and found that those with type 2 diabetes had reduced serum TOC compared with normal glucose and impaired fasting glucose groups (6). Zhou et al. reported lower serum TOC levels in 254 men aged 23-76 years with newly diagnosed type 2 diabetes compared with 126 men with normal glucose tolerance (8). Kindblom et al. studied 1010 elderly men (mean age 75.3 years), and reported lower plasma TOC in men with diabetes and inverse relationships between TOC level with BMI, fat mass, and plasma glucose (7). However, those studies did not examine the relationship between TOC and metabolic syndrome. Our results are consistent with Kindblom et al., as we found that reduced circulating TOC was associated with higher fasting glucose and with greater insulin resistance (HOMA-IR). Our findings are also in keeping with studies restricted to men and women with diabetes. In a study of 149 postmenopausal women (mean age 66.7 years) and 179 men (mean age 64.9 years) with type 2 diabetes, TOC was negatively correlated with fasting glucose and HbAlc (9). Additionally, in a study of 380 adults with diabetes (mean age 71 years), Pittas et al. demonstrated that serum TOC was inversely associated with fasting glucose and HOMA-IR (11). Therefore, our findings illuminate an association between reduced serum TOC with metabolic syndrome in older men, supporting the relevance of osteocalcin to glucose metabolism in humans.

The associations between serum TOC and waist circumference, glucose, and triglyceride levels were most apparent at the levels of TOC below a threshold of $20 \mu \mathrm{g} / \mathrm{l}$. Conversely, there was limited evidence of associations of these variables with serum TOC levels $>30 \mu \mathrm{g} / \mathrm{l}$. In keeping with these observations, we found no apparent linear dose-response gradient for risk of metabolic syndrome with decreasing serum TOC. Instead, men with serum TOC $\leq 16.6 \mu \mathrm{g} / \mathrm{l}$ had increased OR of metabolic syndrome consistent with a 'threshold effect' (27). The OR for metabolic syndrome 

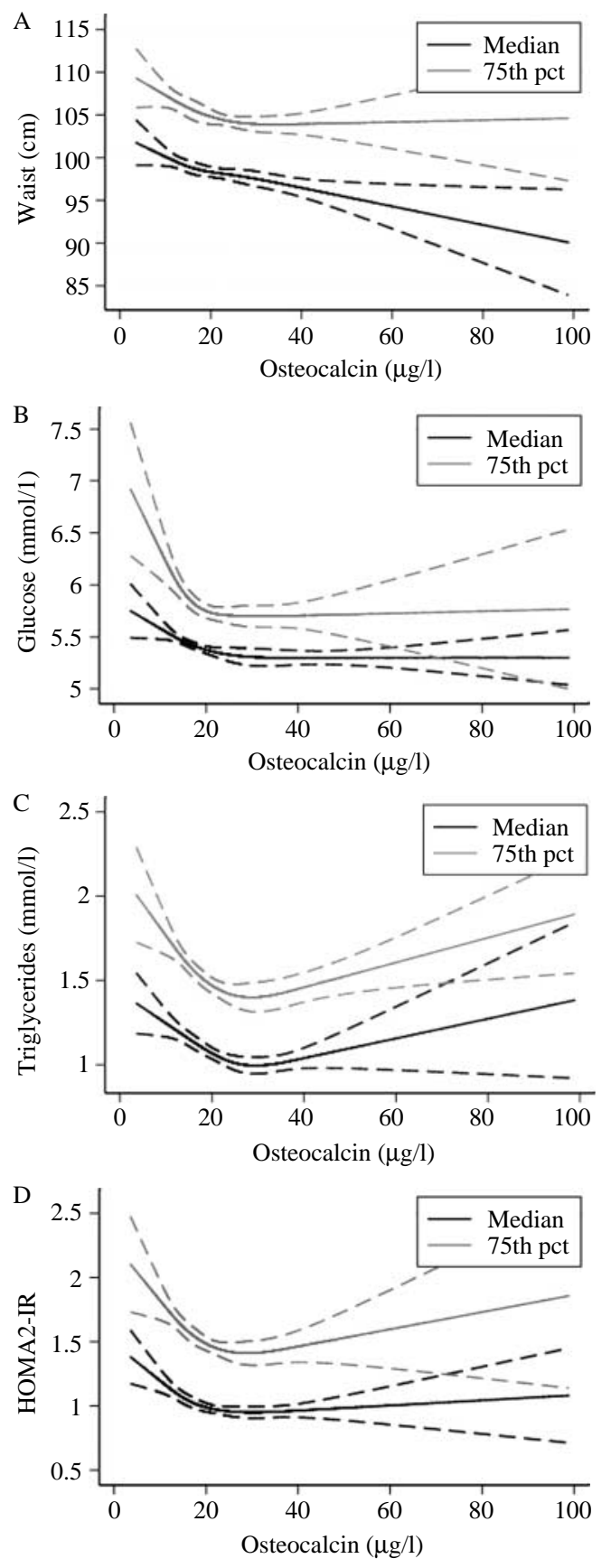

Figure 3 Associations of waist circumference, glucose, and triglyceride levels with serum total osteocalcin. Quantile regression of median and 75th percentile of waist circumference (A), glucose concentration (B), and triglyceride levels (C) against serum total osteocalcin in 2765 older men. Dotted lines are 95\% confidence intervals for the median and 75th percentile respectively. Comparable regression for insulin resistance estimated as HOMA2-IR against serum total osteocalcin (D).

was relatively constant for men with serum TOC levels in the top half of the distribution. There are potential implications of the non-linear association between serum TOC with metabolic syndrome and its individual components. Thus, while reduced serum TOC identifies men at increased risk of metabolic syndrome, higher than average values might not be associated with metabolic benefit.

Strengths of this study include the large size of the cohort, the fact that men were community dwelling and not selected on the basis of any particular condition, the use of early morning sera to minimize any effect of diurnal variation on TOC levels, and the storage of sera at $-80{ }^{\circ} \mathrm{C}$ until assay. Furthermore, we used a TOC assay that is robust for stored (frozen) samples, which detects both intact osteocalcin and the major N-mid terminal fragment (28). This is preferable to an assay for intact osteocalcin, which might underestimate osteocalcin levels due to lability of the C-terminal sequence. We excluded men who had conditions known to affect osteocalcin levels namely bone fracture, Paget's disease, and use of bisphosphonate or glucocorticoid therapy. As warfarin therapy could alter the proportions of undercarboxylated and carboxylated osteocalcin, these men were also excluded from the analysis.

Serum TOC comprises both ucOC and carboxylated osteocalcin. The question of whether ucOC is the metabolically active form of osteocalcin in humans remains to be answered. In a study of 348 non-diabetic men and women aged 68 years, Shea et al. found no association between circulating UcOC and HOMA-IR at baseline or follow-up (29). By contrast, Hwang et al. studied 199 men aged 47 years and reported higher $\beta$-cell function (estimated using HOMA) in men with ucOC levels in the highest tertile of values (30). It is unclear whether smaller sample sizes and the lack of a robust automated assay for ucOC might have contributed to these contrasting results. As we did not have the opportunity to measure ucOC levels, we are unable to clarify this issue. Our findings demonstrate an association between bone turnover and metabolic syndrome in humans, but additional research is needed to determine whether this association is mediated specifically via ucOC distinct from carboxylated osteocalcin. Other limitations of our study are the

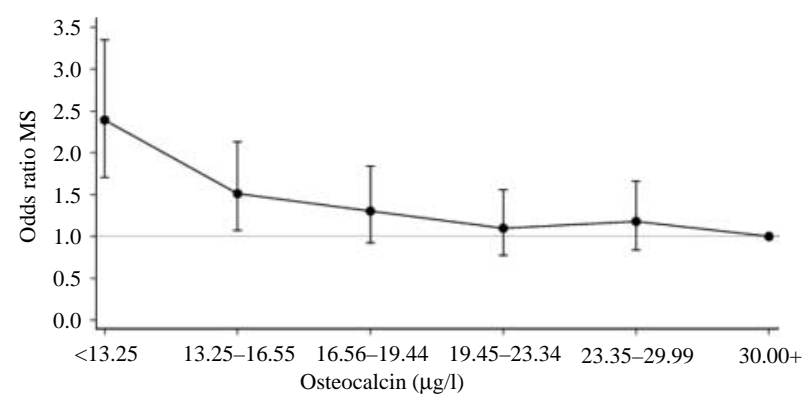

Figure 4 Serum total osteocalcin and metabolic syndrome in older men. Logistic regression analysis adjusted for age, smoking, alcohol use, and serum creatinine in 2765 older men, showing the odds ratio of metabolic syndrome (MS) by serum total osteocalcin, relative to osteocalcin level $\geq 30 \mu \mathrm{g} / \mathrm{l}$. 


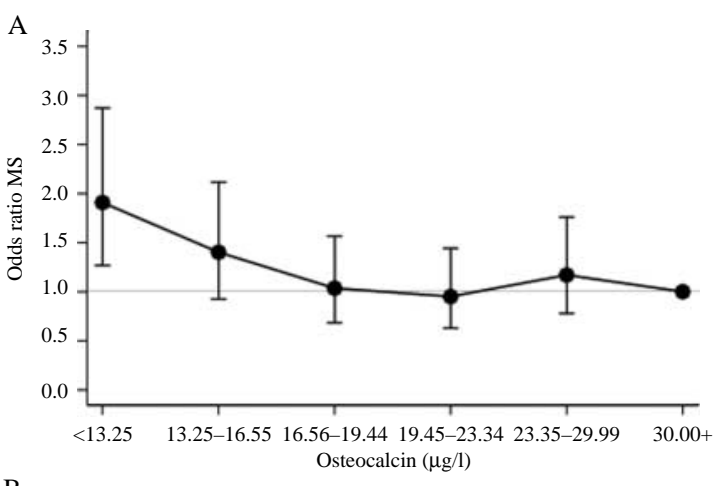

B
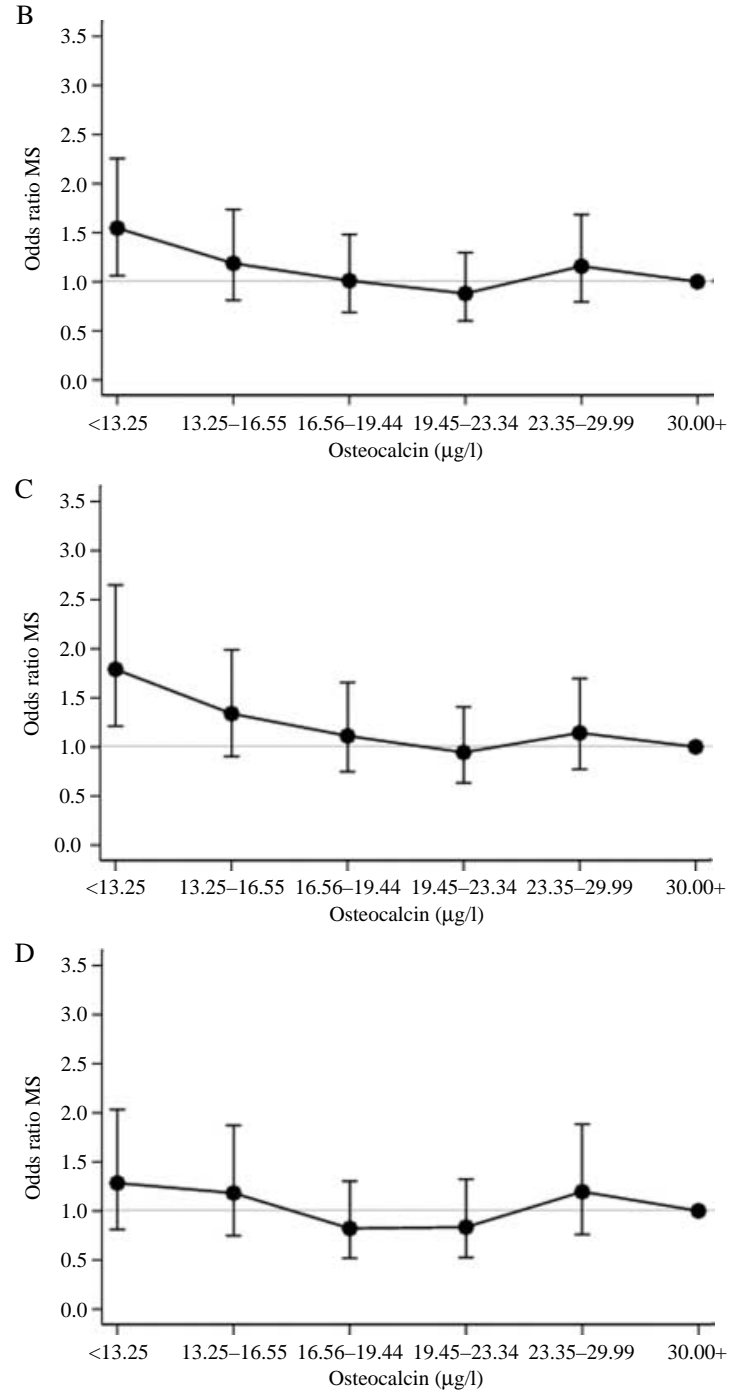

Figure 5 Association between serum total osteocalcin and metabolic syndrome in older men, adjusting for individual components of metabolic syndrome. Logistic regression analysis in 2765 men aged $\geq 70$ years, showing the odds ratio of metabolic syndrome (MS) by serum total osteocalcin, relative to osteocalcin level $\geq 30 \mu \mathrm{g} / \mathrm{l}$, (A) adjusted for baseline covariates (age, smoking, alcohol use, and serum creatinine) and for waist circumference, (B) adjusted for baseline covariates and glucose, (C) adjusted for baseline covariates and triglyceride level, and (D) adjusted for baseline covariates, waist circumference, and glucose. cross-sectional nature of the analysis, which precludes conclusions as to direction of causality. As the inverse association between HOMA2-IR and serum TOC was attenuated in men without metabolic syndrome, this did not suggest a healthy survivor effect. Our cohort comprised community-dwelling older men, thus we cannot comment on associations in women.

Reduced serum TOC was associated with increased odds of metabolic syndrome in our cohort of older men, and this relationship remained significant after adjustment for glucose level. However, the association between serum TOC and metabolic syndrome was abrogated by simultaneous adjustment for waist circumference and glucose concentration. This observation would be consistent with reduced osteocalcin, and elevated waist circumference and glucose levels being related to a common underlying factor, such as insulin resistance. Another plausible explanation would be that these variables lie in a causal pathway, such that reduced osteocalcin level is an intermediate between central adiposity (elevated waist circumference), and insulin resistance manifesting as hyperglycemia. In obese individuals, adipose tissue drives insulin resistance by the release of non-esterified fatty acids, glycerol, hormones such as leptin, and proinflammatory cytokines, with a reduction in adiponectin (for review see (31)). In the setting of insulin resistance, impairment of $\beta$-cell insulin release leads to progressive hyperglycemia and overt diabetes. Of interest, in mice, adipose tissue release of leptin modulates sympathetic nervous system activity and bioavailability of osteocalcin, thus regulating insulin release and sensitivity $(32$, 33). Thus, reduced circulating osteocalcin may function as an endocrine factor, which links central adiposity to insulin resistance and the metabolic syndrome. Whilst our data in men are consistent with this model, specific mechanistic studies would be needed to explore this concept further.

There is a modest age-related reduction in circulating TOC from early adulthood to a nadir in men around the age of 65 years followed by stable levels in older age (34). Insulin resistance, hyperglycemia, and diabetes are predictors of cardiovascular events, as is the presence of the metabolic syndrome (13-16, 24, 25). Men with metabolic syndrome experience an approximate doubling of cardiovascular disease risk (25), and metabolic syndrome is also associated with preclinical atherosclerosis measured by carotid intima-media thickness in men and women (35). Our findings identify reduced serum TOC as a risk predictor for metabolic syndrome in older men, raising the possibility that circulating osteocalcin might modulate cardiovascular risk. However, further investigation would be required to determine whether osteocalcin represents a biomarker or a potential causal factor in this context, and to explore whether interventions targeting men with reduced TOC levels might improve health outcomes. 


\section{Conclusions}

In community-dwelling older men, reduced serum TOC is associated with increased risk of metabolic syndrome. This association reflects the relationship between reduced TOC levels and elevated waist circumference, glucose, and triglyceride levels. Further research is required to clarify the direction of causality and determine whether interventions which raise osteocalcin levels could improve glucose metabolism and decrease cardiovascular risk in ageing men.

\section{Declaration of interest}

The authors declare that there is no conflict of interest that could be perceived as prejudicing the impartiality of the research reported.

\section{Funding}

Hormone assays were funded by research grants from the Fremantle Hospital Medical Research Foundation, Fremantle Hospital, Western Australia, and the Ada Bartholomew Medical Research Trust, University of Western Australia. The Health In Men Study was funded by Project Grants 279408, 379600, 403963, and 513823 from the National Health and Medical Research Council of Australia (NHMRC). B B Yeap is recipient of a Clinical Investigator Award from the Sylvia and Charles Viertel Charitable Foundation, New South Wales, Australia. P E Norman is supported by NHMRC Practitioner Fellowship 458505.

\section{Acknowledgements}

Support from Dr George Koumantakis of Roche Diagnostics Australia for the supply of assay reagents is gratefully acknowledged. The authors thank Johnson Setoh and Seamus Duffy for their excellent technical assistance. We thank the staff and management of Shenton Park Hospital for providing space in which to conduct study-related clinics. We especially thank all the men and staff who participated in the Western Australian Abdominal Aortic Aneurysm Program and the Health In Men Study.

\section{References}

1 Hauschka PV, Lian JB, Cole DE \& Gundberg CM. Osteocalcin and matrix Gla protein: vitamin K-dependent proteins in bone. Physiological Reviews 198969 990-1047.

2 Gundberg CM, Nieman SD, Abrams S \& Rosen H. Vitamin K status and bone health: an analysis of methods for determination of undercarboxylated osteocalcin. Journal of Clinical Endocrinology and Metabolism 199883 3258-3266.

3 Eastell R \& Ebeling PR. Bone turnover markers: a key tool for understanding osteoporosis. Osteoporosis International 200920 S237-S238.

4 Lee NK, Sowa H, Hinoi E, Ferron M, Ahn JD, Confavreux C, Dacquin R, Mee PJ, McKee MD, Jung DY, Zhang Z, Kim JK, Mauvais-Jarvis F, Ducy P \& Karsenty G. Endocrine regulation of energy metabolism by the skeleton. Cell 2007130 456-469.

5 Ferron M, Hinoi E, Karsenty G \& Ducy P. Osteocalcin differentially regulates $\beta$ cell and adipocyte gene expression and affects the development of metabolic diseases in wild-type mice. PNAS 2008 105 5266-5270.

6 Im J-A, Yu B-P, Jeon JY \& Kim S-H. Relationship between osteocalcin and glucose metabolism. Clinica Chimica Acta 2008 396 66-69.
7 Kindblom JM, Ohlsson C, Ljunggren O, Karlsson MK, Tivesten A, Smith U \& Mellstrom D. Plasma osteocalcin is inversely related to fat mass and plasma glucose in elderly Swedish men. Journal of Bone and Mineral Research 200924 785-791.

8 Zhou M, Ma X, Li H, Pan X, Tang J, Gao Y, Hou X, Lu H, Bao Y \& Jia W. Serum osteocalcin concentrations in relation to glucose and lipid metabolism in Chinese individuals. European Journal of Endocrinology $2009161723-729$.

9 Kanazawa I, Yamaguchi T, Yamamoto M, Yamauchi M, Kurioka S, Yano S \& Sugimoto T. Serum osteocalcin level is associated with glucose metabolism and atherosclerosis parameters in type 2 diabetes. Journal of Clinical Endocrinology and Metabolism 200994 45-49.

10 Fernandez-Real JM, Izquierdo M, Ortega F, Gorostiaga E, Gomez-Ambrosi J, Moreno-Navarrete JM, Fruhbeck G, Martinez C, Idoate F, Salvador J, Forga L, Ricart W \& Ibanez J. The relationship of serum osteocalcin to insulin secretion, sensitivity, and disposal with hypocaloric diet and resistance training. Journal of Clinical Endocrinology and Metabolism 200994 237-245.

11 Pittas AG, Harris SS, Eliades M, Stark P \& Dawson-Hughes B. Association between serum osteocalcin and markers of metabolic phenotype. Journal of Clinical Endocrinology and Metabolism 2009 $94827-832$.

12 Vestergaard P. Discrepancies in bone mineral density and fracture risk in patients with type 1 and type 2 diabetes - a meta-analysis. Osteoporosis International 200718 427-444.

13 Khaw K-T, Wareham N, Luben R, Bingham S, Oakes S, Welch A \& Day N. Glycated haemoglobin, diabetes, and mortality in men in Norfolk cohort of European Prospective Investigation of Cancer and Nutrition (EPIC-Norfolk). BMJ 2001322 1-6.

14 Sorkin JD, Muller DC, Fleg JL \& Andres R. The relation of fasting and 2-h postchallenge plasma glucose concentrations to mortality. Diabetes Care 200528 2626-2632.

15 Bonora E, Kiechl S, Willeit J, Oberhollenzer F, Egger G, Meigs JB, Bonadonna RC \& Muggeo M. Insulin resistance as estimated by homeostasis model assessment predicts incident symptomatic cardiovascular disease in Caucasian subjects from the general population. Diabetes Care 200730 318-324.

16 Saely CH, Aczel S, Marte T, Langer P, Hoefle G \& Drexel H. The metabolic syndrome, insulin resistance, and cardiovascular risk in diabetic and nondiabetic patients. Journal of Clinical Endocrinology and Metabolism 200590 5698-5703.

17 Moayyeri A, Kaptoge S, Luben RN, Wareham NJ, Bingham S, Reeve J \& Khaw KT. Estimation of absolute fracture risk among middle-aged and older men and women: the EPIC-Norfolk population cohort study. European Journal of Epidemiology 2009 24 259-266.

18 Norman PE, Flicker L, Almeida OP, Hankey GJ, Hyde Z \& Jamrozik K. Cohort Profile: The Health In Men Study (HIMS). International Journal of Epidemiology 200938 48-52.

19 Expert Panel on Detection, Evaluation, and Treatment of High Blood Cholesterol in Adults. Executive summary of the Third Report of the National Cholesterol Education Program (NCEP) Expert Panel on Detection, Evaluation, and Treatment of High Blood Cholesterol in Adults (Adult Treatment Panel III). Journal of the American Medical Association 2001285 $2486-2497$.

20 Alberti KGMM, Zimmet P \& Shaw J. Metabolic syndrome - a new world-wide definition. A consensus statement from the International Diabetes Federation. Diabetic Medicine 200623 469-480.

21 Wallace TM, Levy JC \& Matthews DR. Use and abuse of HOMA modelling. Diabetes Care 200427 1487-1495.

22 Lee NK \& Karsenty G. Reciprocal regulation of bone and energy metabolism. Trends in Endocrinology and Metabolism 200819 161-166.

23 Kahn SE, Zraika S, Utzschneider KM \& Hull RL. The beta cell lesion in type 2 diabetes: there has to be a primary functional abnormality. Diabetologia 200952 1003-1012. 
24 Wilson PW, D’Agostino RB, Parise H, Sullivan L \& Meigs JB. Metabolic syndrome as a precursor of cardiovascular disease and type 2 diabetes mellitus. Circulation 2005112 3066-3072.

25 Cornier M-A, Dabelea D, Hernandez TL, Lindstrom RC, Steig AJ, Stob NR, Van Pelt RE, Wang H \& Eckel RH. The metabolic syndrome. Endocrine Reviews 200829 777-822.

26 de Rooij SR, Dekker JM, Kozakova M, Mitrakou A, Melander O, Gabriel R, Guidone C, Hojlund K, Murphy MS, Nijpels G \& the RISC Group Investigators . Fasting insulin has a stronger association with an adverse cardiometabolic risk profile than insulin resistance: the RISC study. European Journal of Endocrinology 2009161 223-230.

27 Rothman KJ. Epidemiology: An Introduction, edn 1, ch. 2, pp 8-23. New York: Oxford University Press, 2002.

28 Takahashi M, Kushida K, Nagano A \& Inoue T. Comparison of the analytical and clinical performance characteristics of an N-MID versus an intact osteocalcin immunoradiometric assay. Clinica Chimica Acta 2000294 67-76.

29 Shea MK, Gundberg CM, Meigs JB, Dallal GE, Saltzman E, Yoshida M, Jacques PF \& Booth SL. $\gamma$-Carboxylation of osteocalcin and insulin resistance in older men and women. American Journal of Clinical Nutrition 200990 1230-1235.

30 Hwang Y-C, Jeong I-K, Ahn KJ \& Chung HY. The uncarboxylated form of osteocalcin is associated with improved glucose tolerance and enhanced $\beta$-cell function in middle-aged male subjects. Diabetes/Metabolism Research and Reviews 200925 768-772.

31 Kahn SE, Hull RL \& Utzschneider KM. Mechanisms linking besity to insulin resistance and type 2 diabetes. Nature $2006 \mathbf{4 4 4}$ 840-846.

32 Hinoi E, Gao N, Jung DY, Yadav V, Yoshizawa T, Myers MG, Chua SC, Kim JK, Kaestner KH \& Karsenty G. The sympathetic tone mediates leptin's inhibition of insulin secretion by modulating osteocalcin bioactivity. Journal of Cell Biology 2008 183 1235-1242.

33 Kawai M, Devlin MJ \& Rosen CJ. Fat targets for skeletal health. Nature Reviews. Rheumatology 20095 365-371.

34 Leder BZ, Araujo AB, Travison TG \& McKinlay JB. Racial and ethnic differences in bone turnover markers. Journal of Clinical Endocrinology and Metabolism 200792 3453-3457.

35 Adolphe A, Cook LS \& Huang X. A cross-sectional study of intima-media thickness, ethnicity, metabolic syndrome, and cardiovascular risk in 2268 study participants. Mayo Clinic Proceedings $200984221-228$.

Received 26 April 2010

Accepted 25 May 2010 